\title{
Optical Control over Surface-Plasmon-Polariton-Assisted THz Transmission through a Slit Aperture
}

\author{
E. Hendry, ${ }^{1, *}$ F. J. Garcia-Vidal, ${ }^{2}$ L. Martin-Moreno, ${ }^{3}$ J. Gómez Rivas, ${ }^{4}$ M. Bonn, ${ }^{4}$ A. P. Hibbins, ${ }^{1}$ and M. J. Lockyear ${ }^{1}$ \\ ${ }^{1}$ School of Physics, University of Exeter, Stocker Road, Exeter, EX4 4QL, United Kingdom \\ ${ }^{2}$ Departamento de Física Teórica de la Materia Condensada, Universidad Autónoma de Madrid, E-28049 Madrid, Spain \\ ${ }^{3}$ Departamento de Física de la Materia Condensada, ICMA-CSIC, Universidad de Zaragoza, E-50009 Zaragoza, Spain \\ ${ }^{4}$ FOM Institute for Atomic and Molecular Physics, Kruislaan 407, 1098 SJ Amsterdam, The Netherlands \\ (Received 29 August 2007; revised manuscript received 10 December 2007; published 24 March 2008)
}

We demonstrate optical control over the transmission of terahertz $(\mathrm{THz})$ radiation through a single subwavelength slit in an otherwise opaque silicon wafer. The addition of periodic corrugation on each side of the wafer allows coupling to surface plasmon polaritons, so that light not impinging directly on the slit can contribute to the transmission. A significant enhancement of the $\mathrm{THz}$ transmission can be achieved through control of the surface wave propagation length by excitation at optical wavelengths. The observed transmission increase is in distinct contrast to the reduction reported for photoexcitation of arrays of holes in semiconductors.

DOI: 10.1103/PhysRevLett.100.123901

Stimulated by the seminal work of Ebbesen and coworkers [1], there is renewed interest in the optical properties of subwavelength slits in conductors (see Refs. [2,3] and references therein). A subwavelength slit in a conducting slab is known to support Fabry-Perot-like modes for light polarized perpendicular to the slit [4], giving rise to high transmission when the slab thickness is approximately equal to an integer number of half wavelengths. In addition to Fabry-Perot modes, electromagnetic surface waves (surface plasmons at visible frequencies [5]) also play a key role in determining the transmission of a single slit, in particular, in the presence of ordered surface corrugation at the entrance and exit interfaces. First reported at optical frequencies [2,6], a slit or hole can be made more transmissive by patterning the entrance and/or exit interfaces of the aperture. Similar effects have subsequently been demonstrated at microwave $[7,8]$ and terahertz $(\mathrm{THz})$ frequencies $[9,10]$. The transmission enhancement has been attributed to diffractive coupling of incident light to surface waves, defined by wave vectors parallel to the entrance and exit interfaces, allowing light that does not directly impinge upon the aperture to contribute to transmission. However, due to the different mechanisms which can give rise to peaks in transmission spectra for such structures [2], it is often difficult to differentiate Fabry-Perottype transmission features from effects due to surface waves.

Here, we are able to modulate the low frequency, $\mathrm{THz}$ transmission of a semiconductor structure consisting of a slit in a silicon wafer surrounded by periodic corrugations by optically modifying the propagation lengths of surface waves at the wafer interfaces. This allows us to directly differentiate transmission resonances due to Fabry-Perot modes and surface wave excitation. In the $\mathrm{THz}$ frequency range $(0.2-2 \mathrm{THz})$, all-optical reduction of $\mathrm{THz}$ transmission has been demonstrated for several structures [11-15].
PACS numbers: 42.79.Ag, 42.25.Fx, 73.20.Mf, 78.47.-p

Here, we show how all-optical enhancement of $\mathrm{THz}$ surface modes can increase $\mathrm{THz}$ transmission of a resonant structure, with enhancements approaching a factor of 3 .

The propagation length $L$ of a surface wave propagating at the flat interface of a conductor and vacuum can vary over orders of magnitude depending on the complex dielectric function $\varepsilon$ of the conductor, as is evident from the relation: $L=c / 2 \omega \operatorname{Im}(\sqrt{\varepsilon /(1+\varepsilon)})$ [5], where $\omega$ is the radial frequency. For semiconductors in the $\mathrm{THz}$ frequency range, the dielectric function can be approximated using the Drude model:

$$
\varepsilon(\omega)=\varepsilon_{\text {undoped }}-\frac{\omega_{p}^{2}}{\omega^{2}+i \omega \gamma},
$$

where $\varepsilon_{\text {undoped }}$ is the dielectric constant of the undoped semiconductor, $\omega_{p}{ }^{2}=e^{2} N /\left(\varepsilon_{0} m^{*}\right)$ is the plasma frequency (dependent on the density of charge carriers $N$, and carrier effective mass $m^{*}$ ) and $\gamma$ is the charge scattering rate. By modifying $N, L$ is tunable over a very large range in the THz region: Fig. 1(a) depicts $L$ as a function of the Drude parameters for $\omega / 2 \pi=1 \mathrm{THz}$. For the $p$-doped silicon wafers investigated here (360 $\mu \mathrm{m}$ thick from Wafer World, conductivity $\sim 1500 \mathrm{~S} / \mathrm{m} ; \varepsilon_{\text {undoped }}=11.8 ; \gamma \sim$ $15 \mathrm{THz}$ and $\left.\omega_{p} \sim 60 \mathrm{THz}[16]\right) L \sim 1 \mathrm{~mm}$. By photodoping with a visible frequency light pulse, the density of free charges can be increased by orders of magnitude, causing a large increase in $\omega_{p}$ [17] and $\gamma$ [18] in the nearsurface region, and inducing a large increase in the propagation length of surface waves [in Fig. 1(a)]. Indeed, $L$ may approach the values expected for metals at $\mathrm{THz}$ frequencies [19]: for a reasonable optical fluence of $1.5 \times$ $10^{26}$ photons $/ \mathrm{m}^{2}$ we estimate from [17] that $L \sim 1 \mathrm{~cm}$.

Samples were designed to highlight this tunable property. Sample A is formed from a $360 \mu \mathrm{m}$ thick $p$-doped silicon sample into which we cut a $40 \mu \mathrm{m}$ wide slit aper- 

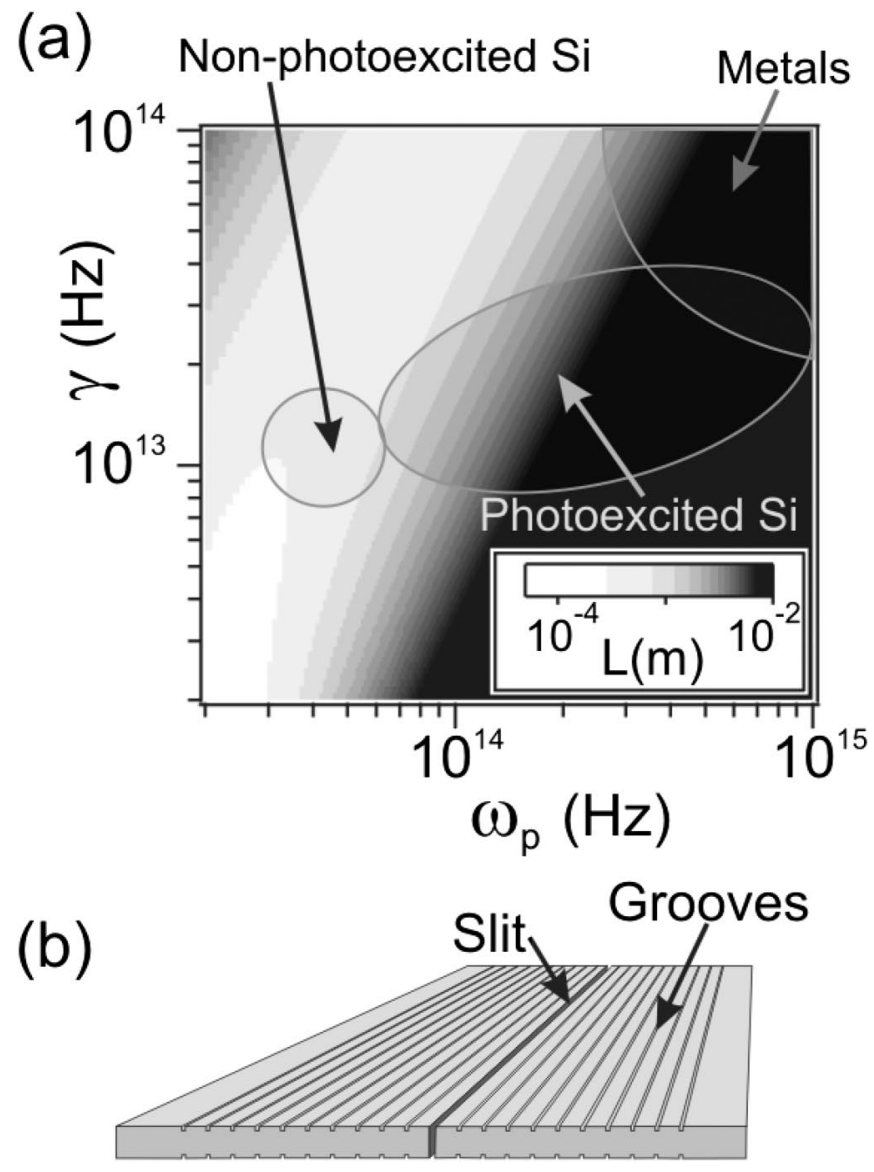

FIG. 1. (a) Propagation length (in $\mathrm{m}$ ) of a $1 \mathrm{THz}$ surface mode on a flat silicon interface, as a function of plasma frequency and scattering rate. On photoexcitation we can approach propagation lengths of metals [22]. (b) Illustration of sample B.

ture using a diamond edged wafer saw. Sample B is identical to sample A with the addition of grooves of $40 \mu \mathrm{m}$ width and $60 \mu \mathrm{m}$ depth, spaced by $500 \mu \mathrm{m}$, on both entrance and exit sides of the slit [see Fig. 1(b)]. Grooves allow for diffractive coupling to surface waves, similar to the concentric grooved "bullseye" structure reported previously [6]. The groove dimensions were optimized using analytical modeling [2]. We note that the surface wave propagation lengths for sample $\mathrm{B}$ are expected to be shorter than shown in Fig. 1(a) due to scattering by the grooves.

We measure the zero order $\mathrm{THz}$ transmission [13] of the samples with a $\sim 5 \mathrm{~mm}$ diameter beam polarized perpendicular to the slit. The broadband $\mathrm{THz}$ pulses are essentially single cycles of the electric field of about $1 \mathrm{ps}$ duration-see inset of Fig. 2(a). By Fourier transforming the time domain waveforms measured with and without sample $\left[E(t)\right.$ and $E_{0}(t)$, respectively] the intensity transmission spectrum, $|E(\omega)|^{2} /\left|E_{0}(\omega)\right|^{2}$, is readily calculated. The structures are photoexcited using $150 \mathrm{fs}, 800 \mathrm{~nm}$ laser pulses to generate free carriers in the near-surface region [17]. The transmission changes were measured at a delay between excitation and $\mathrm{THz}$ probe pulses of $10 \mathrm{ps}$, to allow
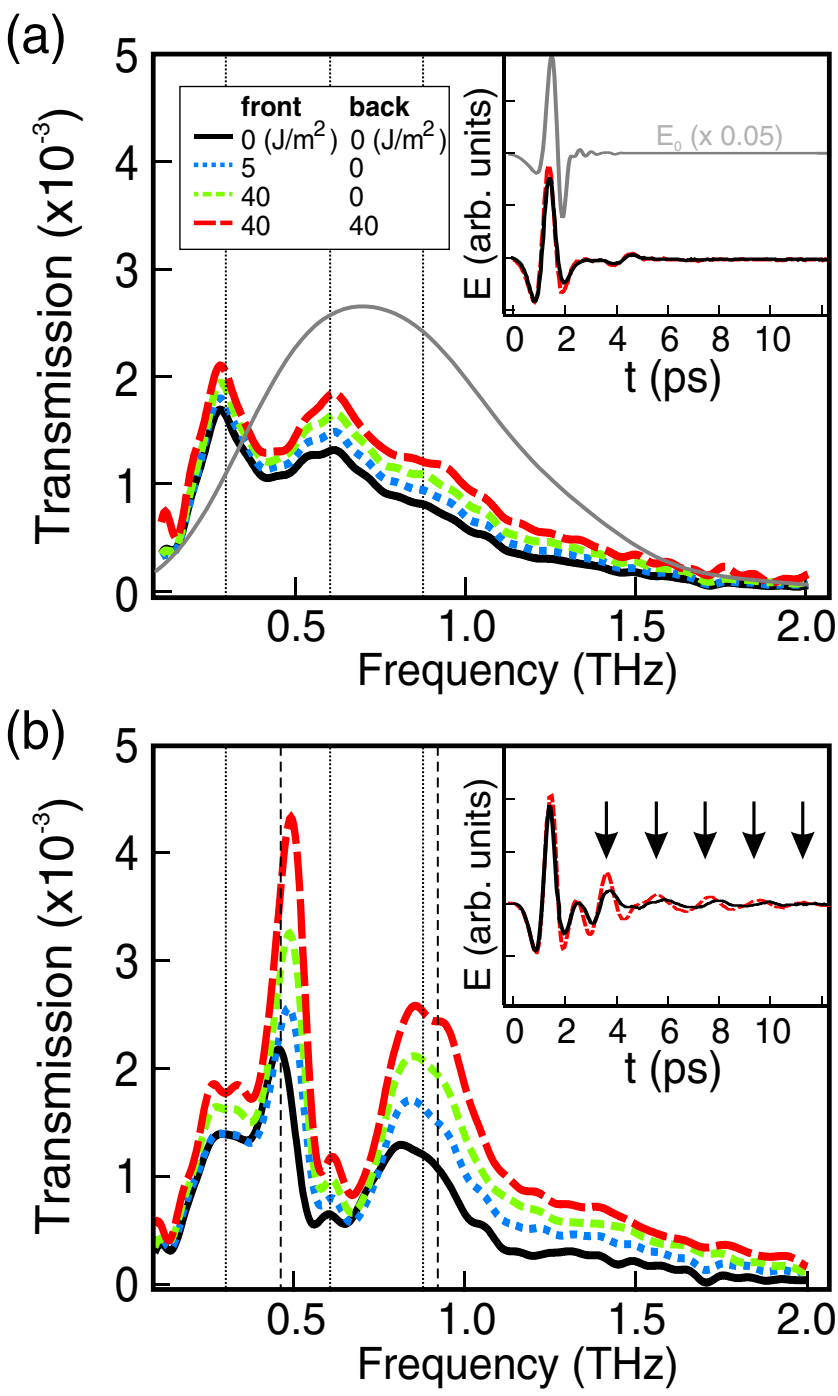

FIG. 2 (color online). (a) Transmission of sample A for various $800 \mathrm{~nm}$ photoexcitation intensities of the front and back interfaces. The thin gray curve shows the scaled spectrum of the reference $\mathrm{THz}$ pulse. (b) Transmission of sample B (grooved sample). Dotted and dashed vertical lines denote Fabry-Perot and surface resonances, respectively. Insets: Time domain field transmission with [red (or gray) dashed] and without (black full) visible frequency excitation. In the inset of the upper panel, the scaled time domain reference pulse is offset for comparison. In the inset of the lower panel, the arrows mark the sequential contributions from diffraction from the 1st, 2nd, 3rd, 4th, and 5th pairs of grooves subsequent from the slit aperture in sample B.

for carrier cooling without loosing carriers due to diffusion or recombination $[13,18]$. The back and front interfaces of the structure can be photoexcited independently or simultaneously with separate laser pulses, modulating the propagation length of surface modes on either or both interfaces.

With no grooves (sample A), the transmitted $\mathrm{THz}$ pulse in the time domain contains a recurrence 3 ps after the main pulse [see inset of Fig. 2(a)] due to reflection within the slit cavity. The transmission spectrum reveals weak 
resonances due to this Fabry-Perot like reflection at $\sim 0.3$, 0.6 , and $0.85 \mathrm{THz}$ [vertical lines in Fig. 2(a)]. These modes are broad and significantly redshifted from the resonant frequencies expected for a highly conductive metal due to the finite slit width [4] and losses in the poorly conducting silicon [20]. Upon photoexcitation of both the front and back interfaces, a small increase in the transmission is observed, attributed to photoinduced variations in the coupling between the incident radiation and Fabry-Perot modes inside the slit.

For the grooved sample (sample B), in the time domain we observe ringing after the main pulse with a period defined by the groove period [arrows in inset of Fig. 2(b)]. These sequential recurrences originate from $\mathrm{THz}$ light scattered by grooves increasingly far from the slit; the delay is caused by the time it takes for the scattered waves to propagate along the surface towards the slit [10]. In the transmission spectrum [black solid line in Fig. 2(b)] the Fabry-Perot modes are still visible, while two new features emerge at $\sim 0.45 \mathrm{THz}$ and $\sim 0.9 \mathrm{THz}$ (vertical dashed lines). Photoexcitation results in a strong enhancement and slight blueshift of these new features.

We attribute the $\sim 0.45$ and $\sim 0.9 \mathrm{THz}$ peaks to scattering from the grooves on the incident face of the sample, allowing $\mathrm{THz}$ light that does not directly impinge upon the slit to contribute to transmission. At these frequencies, the groove separation is approximately one and two wavelengths, respectively, so that surface waves on this interface will constructively interfere at the slit entrance. On photoexcitation, longer propagation lengths of surface waves are realized and more light is scattered into the slit, leading to an enhancement of the transmission. Moreover, the scattering losses of the surface waves themselves are diminished on photoexcitation owing to the reduced evanescent decay length of the electric field into the silicon [21]. The apparent increase in transmission upon photoexciting the back interface can be understood by noting that, although a grating on the output side cannot "pull" more light through an aperture [2,9], it can result in an increased directionality ("beaming") of the exiting light [6]. Hence, photoexciting the back interface of sample B results in the directing of $\mathrm{THz}$ light towards our detector.

To confirm the assignment of the transmission features at $\sim 0.45$ and $\sim 0.9 \mathrm{THz}$, we have carried out finite element method modeling (HFSS, Ansoft) assuming a dielectric function for our silicon from Eq. (1) with $\gamma \sim 15 \mathrm{THz}$ and $\omega_{p} \sim 60 \mathrm{THz}[16]$. We approximate the photoexcited surfaces of sample B as perfect conductors (i.e., with infinite conductivity): for the largest experimental excitation fluences, $\omega_{p}$ at the surface approaches $10^{15} \mathrm{~Hz}$ [17], comparable to that for metals [22]. Note that, in both model and experiment, the region inside the slit remains unexcited. The modeled zero order transmission spectra before (black solid line) and after [red (or gray) dashed line] photoexcitation are plotted in Fig. 3(b), and compared to (a) measured

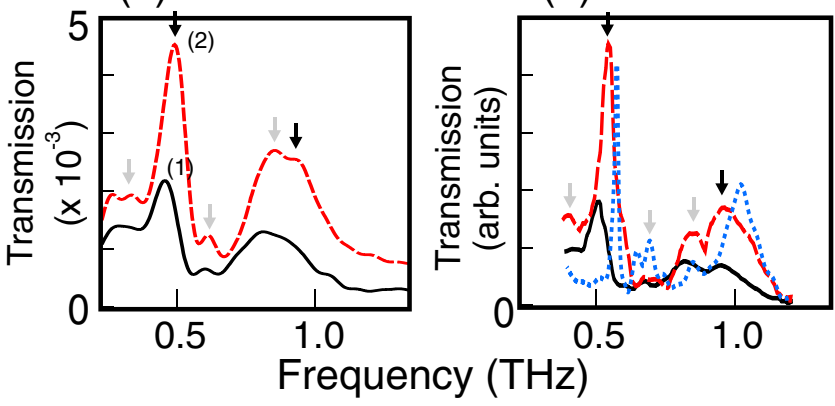

(c) modeled fields
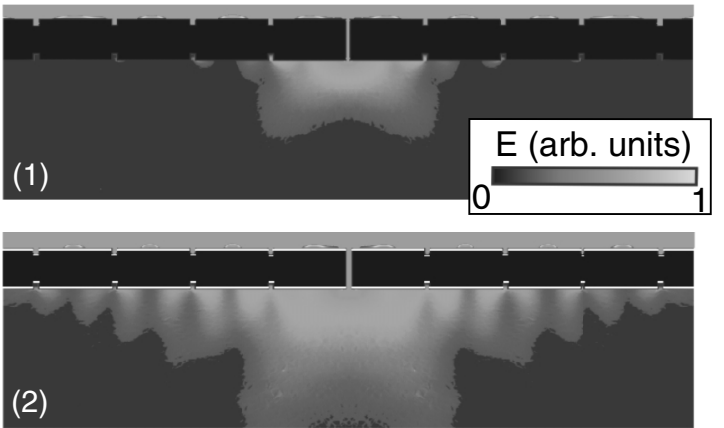

FIG. 3 (color online). (a) Measured transmission spectra as presented in Fig. 2(b). Black and gray arrows indicate the features attributed to the excitation of surface modes and Fabry-Perot reflection, respectively. (b) Modeled transmission spectra for sample B (black line) and the same sample assuming perfectly conducting interfaces at the entrance and exit sides of the slit [red (or gray) dashed line]. The dotted blue (or dark gray) line shows transmission predicted by the analytical model in Ref. [2]. (c) The modeled time-averaged field magnitudes for the peak resonant frequencies $(1,2)$ in $(a)$, assuming a plain propagating $\mathrm{THz}$ wave incident from the top. The doped silicon is shown in black, while the white regions in the lower panel indicate photoexcited (perfectly conducting) surfaces.

the experimental data in Fig. 3(a). The spectra in Fig. 3(b) show the five features observed in the experimental data (indicated by arrows in Fig. 3), resulting from: (1) the excitation of surface modes $(\sim 0.45$ and $\sim 0.9 \mathrm{THz})$, and (2) Fabry-Perot resonances within the slit $(\sim 0.3,0.6$, and $0.85 \mathrm{THz})$. Note that the peaks at $\sim 0.85$ and $\sim 0.9 \mathrm{THz}$ in the measured data are not well resolved due to limited spectral resolution $(\sim 0.1 \mathrm{THz})$. The slight blueshift of resonant frequencies on photoexcitation is also reproduced. There is also a small blueshift for the modeled peaks with respect to measurement, suggesting that the model underestimates losses due to absorption and inhomogeneity in the sample. Nevertheless, despite some significant approximations (e.g., in assuming Drude model and perfect metal dielectric functions), the overall agreement with experiment is good, and the enhancement of the $0.45 \mathrm{THz}$ feature on photoexcitation is particularly well reproduced. In Fig. 3(b) we also compare our finite element modeling to that from the analytical model described in 
Ref. [2] [blue (or dark gray) dotted line], which assumes that sample B is constructed entirely from perfect metal. The resonant peaks in this model are again blueshifted and significantly narrower. This indicates that absorption of $\mathrm{THz}$ light in the unexcited region inside the slit plays an important role in determining the transmission of our sample.

In Fig. 3(c) we plot the modeled time-averaged electric fields at $0.45 \mathrm{THz}$. The influence of photoexcitation on the $\mathrm{THz}$ light emerging from the slit is clear: without photoexcitation, the intensity of surface waves near the outer grooves is very weak, due to their short propagation lengths. Upon photoexcitation, surface waves are able to propagate towards the outer grooves where they can couple to freely propagating light modes. It has been shown previously [9] that increasing the number of corrugation periods on either side of an aperture can increase the intensity of forward transmission. Here, we effectively increase the number of grooves contributing to the transmission without physically altering the structure. According to the modeled fields, the first 5 grooves can affect transmission - this agrees with the number of recurrences observed in the time domain measurements (arrows in the inset of Fig. 2). Our model further reveals that the experimentally observed factor of 2-3 times enhancement on photoexcitation of the $0.45 \mathrm{THz}$ peak may increase to a factor of $\sim 5$, if the wavelength of this peak is matched to the Fabry-Perot mode of the slit [2].

With active optical devices in mind, all-optical switching of low frequency resonant transmission is highly desirable for a variety of applications, as ultrashort laser pulses allow for high $(>\mathrm{GHz})$ modulation frequencies [13]. In contrast to the all-optical reduction of $\mathrm{THz}$ transmission reported for semiconductor hole arrays [11,12], an effect attributed simply to a decrease in the effective cutoff frequency of the apertures on photoexcitation [11], we have demonstrated here an all-optical enhancement of $\mathrm{THz}$ transmission of our structure, consisting of a slit in a silicon wafer surrounded by periodic corrugations.

We thank J.R. Sambles and W. L. Barnes for helpful discussions. E. H., A.P.H., and M. J.L. acknowledge the support of the EPSRC (U.K.). The contribution by M. B. and J.G.R. is part of the research program of the "Stichting voor Fundamenteel Onderzoek der Materie," which is financially supported by NWO. F. J.G. V. and L. M. M. acknowledge the financial support by the Spanish MECD under Contract No. MAT2005-06608-C02.

*e.hendry@exeter.ac.uk

[1] T. W. Ebbesen et al., Nature (London) 391, 667 (1998).

[2] F. J. Garcia-Vidal et al., Phys. Rev. Lett. 90, 213901 (2003).

[3] W. L. Barnes et al., Nature (London) 424, 824 (2003).

[4] Y. Takakura, Phys. Rev. Lett. 86, 5601 (2001).

[5] W. L. Barnes, J. Opt. A Pure Appl. Opt. 8, S87 (2006).

[6] H. J. Lezec et al., Science 297, 820 (2002).

[7] A. P. Hibbins et al., Appl. Phys. Lett. 81, 4661 (2002).

[8] M. J. Lockyear et al., Appl. Phys. Lett. 84, 2040 (2004).

[9] A. Agrawal and A. Nahata, Opt. Express 14, 1973 (2006).

[10] A. Agrawal et al., Opt. Express 13, 3535 (2005).

[11] C. Janke et al., Opt. Lett. 30, 2357 (2005).

[12] W. Zhang et al., Phys. Rev. Lett. 98, 183901 (2007).

[13] E. Hendry et al., Phys. Rev. B 75, 235305 (2007).

[14] H. T. Chen et al., Opt. Lett. 32, 1620 (2007).

[15] L. Fekete et al., Opt. Lett. 32, 680 (2007).

[16] Drude parameters for our wafers are estimated from Hall measurements on a $p$-doped silicon wafer with similar conductivity [see R. Hull, Properties of Crystalline Silicon (INSPEC, London, 1999)]. Conductivity effective mass for holes is taken to be $m^{*} \sim 0.4 m_{e}$.

[17] On short (ps) time scales, expansion or recombination is expected to be negligible. In this case, an estimate for the charge density can be obtained from the excitation fluence $f_{l}$ and the penetration depth of the visible light $l_{0}$ through $N \approx\left(f_{l} / l_{0}\right)$. For $800 \mathrm{~nm}$ excitation $l_{0} \sim 1 \mu \mathrm{m}$. Plasma frequencies can be estimated using $\omega_{p}{ }^{2}=e^{2} N /\left(\varepsilon_{0} m^{*}\right)$, with electron effective mass $m^{*} \sim 0.2 m_{e}$.

[18] E. Hendry et al., Phys. Rev. B 75, 233202 (2007).

[19] K. Wang and D. M. Mittleman, Nature (London) 432, 376 (2004).

[20] J. R. Suckling et al., Phys. Rev. Lett. 92, 147401 (2004).

[21] J. A. Sánchez-Gil and J. G. Rivas, Phys. Rev. B 73, 205410 (2006).

[22] M. A. Ordal et al., Appl. Opt. 22, 1099 (1983). 